\title{
$\bullet$ \\ IJCRR \\ Section: Healthcare \\ Early Detection and Screening of Breast Cancer: An Investigation Case on Mammography Risks in Mauritians
}

Sci. Journal Impact

Factor: 6.1 (2018)

ICV: 90.90 (2018)

(c) (3) (8)

Copyright@IJCRR

\section{Treevedeeta Akshita Parbhudayal ${ }^{1}$, Fatemeh Meskaran², Chandra Reka Ramachandiran ${ }^{3}$}

${ }^{1,2,3}$ Asia Pacific University of Technology and Innovation.

\section{ABSTRACT}

Background: Breast Cancer affects every, one in five women in the world. To date, no cure is available for patients suffering from cancer in their last stage. The only cancer treatment is to diagnose the disease at an early stage and be medically supervised. However, the golden tool used for breast cancer screening, 'Mammography' has been highly debated for the risks it imposes on women of different ages.

Purpose: This research aims to investigate the risks of mammography on women of different ages as well as to suggest a safer alternative to women in Mauritius. The concept of thermal screening is introduced whereby its effectiveness is further explained.

Conclusion: The research also includes a survey and analysis of the perception of women in Mauritius, towards the use of a sensor wearable device for the screening of breast cancer.

Key Words: Breast Cancer, Mammography, Mammography Risks, Thermal Screening, MRI, Sensors

\section{INTRODUCTION}

The study focuses on the risks of mammography screening and the need to find a safer alternative. As much as mammography is considered as the golden tool for breast cancer testing, it happens to have some disadvantages. The problem statement administers the risks of mammography testing on women in Mauritius.

It has been found that the failure rate of breast cancer is rising considerably since most cases are identified in the last stages. ${ }^{1}$ Additionally, more studies have proved that women do not have the accessibility to early breast cancer screening as it is quite costly to afford every year, as well as there, were incidents of failed mammography tests for cancer patients. The study will significantly focus on proposing an advanced sensor-enabled and wearable technological tool, to detect any abnormalities around the breast at an early stage. Furthermore, a quantitative survey will be designed targeting the female population in Mauritius to find their perspective on the device and to evaluate if this can be implemented in the curriculum of the Ministry of Health and Quality of Mauritius. $^{2}$

\section{BACKGROUND STUDY}

Based on the world health organization report, breast cancer is known to be the second deadliest cancer with no cure if diagnosed at a critical stage. It is a universal medical disease affecting only women and more than 1.38 million cases have been detected in 2018, worldwide. Due to the lack of available resources for early-stage testing, in many countries, many patients are diagnosed at a late and critical stage. ${ }^{1}$ This has led to more than 41,000 deaths annually despite medics giving all the required treatments including chemotherapy. Further to this, clinics in Mauritius are equipped with mammography, thermography, ultrasound, MRI which are quite costly to afford every year. There is a high need to introduce a patient-centred application and device, allowing the individual to test themselves at their comfort. This will prove to be a smart, cost-effective, and non-ionic radiated screening tool for the detection of any abnormality. ${ }^{7}$

Mauritius is an island with a population of 1.2 Million. Comparing to the past decade, the life expectancy of the ageing population has increased considerably as people are more

\section{Corresponding Author:}

Treevedeeta Akshita Parbhudayal, Asia Pacific University of Technology and Innovation. Email: Akshita.Parbhudayal@gmail.com

ISSN: 2231-2196 (Print)

Received: 15.08 .2020
ISSN: 0975-5241 (Online)

Revised: 15.09 .2020
Accepted: 22.10 .2020
Published: 24.11 .2020 
healthy. However, since it is still a developing nation, technological advancements are very slow. ${ }^{2}$ Dr. Anwar Husnoo, Ministry of Health and Quality of Life, stated that the treatment for cancer care has increased significantly during the last five years and to be able to cope with the recovery of patients more effectively, the ministry is planning to expand the medical care in clinics by introducing advanced technology to treat patients. It is expected that by 2020 , there will be smart clinics for cancer treatments. Link To Life (Breast Cancer Association of Mauritius) has reported more than 1,500 cases along with 350 deaths from breast cancer patients every year in Mauritius.

Various Studies substantiate the statement that breast cancer is curable if detected at an early stage ${ }^{5}$. Early-stage cancer is treatable, and the medical industry has treated early-stage patients with more than $95 \%$ success rate hence proving to be curable and non-fatal as compared to late-diagnosed cancer. ${ }^{1}$ There are several devices available for breast cancer testing available in clinics and hospitals in Mauritius, however, the services are not always free except on special breast cancer campaigns. It is recommended for women aged 25 and above to conduct breast screening every two years but Mauritius reported a fall in women going for cancer screenings majorly for the cause of being costly to afford every year. Moreover, some women were also skeptical about doing mammography as studies have shown that repeated $\mathrm{x}$-ray can cause radiation exposure hence promoting the growth of cancer cells.

While mammography screening is considered as the ideal device for breast cancer detection, it has also many underlying side-effects which many people and medical institutions tend to ignore. There have been numerous debates on using mammography as a prime breast cancer detection tool. Mammography is conducted by using x-ray beams to capture the insides of a person and to image it in a black and white radiograph. In doing so, $\mathrm{x}$-ray emits ionizing radiation which can cause harm to living tissues. ${ }^{6}$

Mammography screenings have often given inaccurate results of 'false-positive', because of factors such as - Density of breasts and the age of the individual, due to which the latter must be screened again to confirm the result. During this process of over-diagnosing, the person may be exposed to high radiation which may cause harm to them. Many Research Institutes have identified over-diagnosis being a factor to develop breast cancer. The benefits of using the tool are questioned as there are more harms than good. ${ }^{8}$

The price of a mammography screening is also quite hefty to afford every two years. This explains the low participation rate of women for early breast cancer screening in Mauritius. Based on a survey by Link To Life. ${ }^{7}$ The statistics show that only $45 \%$ of women have been screened for early breast cancer, $65 \%$ are aware of the risks of late cancer detection, and $38 \%$ of breast cancer patients have been diagnosed at a late stage. Therefore, women are at risk because of the low accessibility of getting a screening done.

This research identifies the causes of breast cancer formation, the risks of mammography screening towards women of different age are presented in next section, and it recommends a safer alternative to Mammography (Thermal wearable device - Cyracadia Health)

\section{LITERATURE REVIEW}

In the simplest term, Mammography is an e-rayed image of the breast to show any abnormalities in that area. As every woman is different, there are different techniques available for breast cancer screening depending on the seriousness of cancer, age of the women, the density of their breasts, and the availability of the screening devices. ${ }^{8}$ With the advancements in technology, there have been new methods of X-rays namely the Digital Mammography, Computer-aided Detection (CAD), and Breast Tomosynthesis, to have better imaging of the breast.

A biopsy is assisted by a medical practitioner who will set the patient ready for the $\mathrm{x}$-ray. As it is done, a radiologist will interpret the result to find any abnormalities in the breast area. It is considered the best method to diagnose a breast cancer disease for healthy women as in many situations, it reduces the mortality rate of the patient. ${ }^{13}$

As much as it is vital to detect early symptoms of breast cancer, the only possible way to do so in Mauritius is through a Biopsy. To be able to see the development of the disease, there needs to be a continuous, linear increase of the malignant cells, forming a pattern that will allow the radiologist to detect cancer. Thus, if nothing was detected after a biopsy, the use of mammography screening might not be the best choice for primary diagnosis. The World Health Organization recommended medical institutes to follow a ten-rule practice before performing a biopsy because of the risks involved in mammography but, the rules are only adhered to by first world countries due to the wide range of tools available for screening. ${ }^{14}$

However, the subject of effectiveness is highly debatable since many countries have seen a decrease in mortality rate after introducing breast cancer screening for women using Biopsy. The 2012 Health Review of Ireland stated a 26\% reduction in mortality amongst women who participated in a breast screening program and many were followed up by medics throughout 8 to 11 years. Hanley, Hannigan, and O'Brien explain that mammography is harmless since the human body can absorb a capacity of $3 \mathrm{mSv}$ and the radiation emitted by a biopsy is only $0.4 \mathrm{mSv}^{7}$. In his opinion, mammography testing is the best tool for an early screening as it has been used for more than 30 years and there have been 
many technological advancements such as Digital Mammography that has enhanced the results i.e. less 'false-positive results). The advanced digital mammography is designed with low radiation emittance and better visual display, allowing the radiologist to have better accuracy while doing the interpretation. John Hopkins Medicine Institute elucidate on the fact that mammography is not performed straight-away but after following a set of procedure. Before the screening, the patient's breasts are examined by an oncologist and is based on the findings such as the density of breast, age, and genetic history, the appropriate mammography is given to the patient. For instance, screening mammography is performed on an individual showing no symptoms and a Diagnostic Mammogram for unusual breasts or the presence of discomfort, pain, or discharge. ${ }^{11}$

In contrast to the above findings, Komen. $G$ deduces that the accuracy and effectiveness of a Biopsy is only $87 \%$. The method works best for women aged 50 and above as their body is more resistant to the radiation..$^{13}$

\section{FACTORS TRIGGERING BREAST CANCER}

Many cancer institutes around the world, work collaboratively with the World Cancer Research Fund International to identify and analyze the factors triggering breast cancer. Findings by the Union for International Cancer Control (UICC) has identified four factors ${ }^{4}$ causing the multiplying of excessive cells to form cancer including:

\section{Genetic Risk Factors}

It is among the most identified cause of breast cancer. Hereditary accounts $5 \%$ of the diagnosed breast cancer cases which indicates that women with breast cancer family history are mostly prone to develop cancer during a younger age as compared to other women. The World Health Organization has strongly recommended women with cancer family background to perform regular check-ups. In a study by Hannigan genes, BRCA1 and BRCA2 are more likely to develop breast and ovarian cancer. ${ }^{12}$

\section{Heavy Alcohol Consumption}

Women consuming heavy alcohol are liable to several risks of cancer. O'Brien analyses the increase of breast cancer patients in Europe and has identified alcohol consumption and smoking to be one of the major factors. Alcohol forms part of the European Lifestyle and based on his survey (France 2015): $26 \%$ of women drink occasionally, $29 \%$ do not consume alcohol, $45 \%$ drink more once or more per week

As compared to Saudi Arabia, there are lesser cases of women diagnosed with breast cancer through alcohol consumption $-2 \%$ which shows a relationship with breast cancer development and lifestyle. ${ }^{10}$
A similar relationship is identified with tobacco smoking by Kori.S. It does not only promote the development of breast cancer but lung, kidney, and pancreas cancer. The chemical carcinogens in tobacco prove to trigger the mammary receptors and over time, form a tumor. ${ }^{13}$

\section{Exceeding Radiation Exposure}

Radiation Exposure became a potential risk when patients who had been through radiation exposure incidents which happened during the ' 80 s. These patients were closely monitored by a group of scientists in Japan and proved to have serious consequences such as the development of diseases because of several organs failing. ${ }^{9}$

Radiation affects women differently based on their age. Younger women under 20 years face higher risks of radiation-related breast cancer. However, women aged more than 50 have no potential in having an increased risk of breast cancer development through radiation. As women grow older, the process of puberty allows the immunity to strengthen hence become less radiosensitive. Women during their pregnancy are highly radio-sensitive and may cause complications to their new-born. ${ }^{8}$

\section{DNA Alterations}

DNA isa genetic attribute given to us by birth. However, with environmental factors and gene mutations, people start developing unwanted diseases. DNA changes refer to our way of living and the changes that come with time. For example, someone may carry cancerous genes but if she adopts a clean lifestyle - (no smoking or drinking), the person has lesser chances of developing cancer. As stated by Kori.S, cancer grows when there is an accumulation of DNA damage and repair failures. ${ }^{12}$

\section{Overview of the causes of cancer}

There are many causes of breast cancer and scientist has listed the five mentioned cause as the prime concerns. The highlight of the causes according to the researcher's finding is the 'over-diagnosis' of patients. As much as screening is necessary, the result can be achieved but through a different method that does not carry potential risks of harming an individual in the long-term. In 2020, huge Tech Giants are making millions off on mobile health applications by using sensors to track their bodily movement. With the innovations in technology, people are having access to personalized health tools that can be used at their convenience. Nowadays, there are millions of small business mushrooming online providing online doctor, disease diagnosis, drug reference, and many more. As everything is moving towards digitalization and due to its numerous benefits, it will also be valuable if cancer screening is conducted more safely. ${ }^{11}$ 


\section{Mammography Risks}

To date, there are no medicines that can cure any form of cancer. Deadly diseases require special attention and women from the age of 20 are encouraged to conduct regular screenings. However, many studies have proved the relationship with the formation of cancerous cells with mammography, the golden tool for breast cancer detection for women of all ages. The use of radiation has been used during the $1950 \mathrm{~s}$ for internal imaging, and in 2020, with the advancements of technology, new devices of mammography have been introduced such as the digital mammogram. However, the cumulative effect of regular mammography has high risks of forming cancerous cells in young women. Other than this, many risks have been reviewed by researches based on the risks imposed on mammography for women. ${ }^{6}$

The participation rate of women in European and Western Countries (America, Canada, Brazil) tend to be higher than those of some Asian Countries (India, Thailand, Indonesia) with a $70 \%$ against $45 \%$ rate. Mauritius, being also a developing country has been able to provide free health care to its citizens. Breast Cancer Campaigns are organized every six months by Link To Life (Mauritius Breast Cancer Association) where free screenings are provided for women over 35. Besides hospitals, clinics provide annual packages for health screenings which also includes breast screening. It is to be highlighted that most hospitals are equipped with analogue Mammography while clinics use digital mammography.

There are two main mammography devices which are the analogue and the digital. The analogue has many drawbacks because it provides low-quality imaging and in cases of bad imaging, a second x-ray is taken to confirm the biopsy. The digital has been introduced to have better imaging so as there is no need for more $\mathrm{x}$-ray. It is to be highlighted that mammograms emit low radiation but with several screenings, the radiation may cause damage to the cells. ${ }^{12}$ Besides, there are other possible risks including:

\section{False Negative Results}

A False Negative Result happens when a patient is declared Cancer Positive after a biopsy due to the inaccuracy of mammography. The patient will then be undergoing further medical supervision for breast cancer care following the results and may then know that the result was incorrect. In many cases, patients lived with the thought they have breast cancer while in reality, the result was inaccurate. As per the International Medical Norms, it is denied having more than two biopsies in one year. Mammography can only be done once every 2 years interval. Hence, the false-negative result has also caused stress and depression for women as they are under the pressure of mental preparation to overcome the disease. $^{11}$

Sensitivity and accuracy of mammography depend on the density of the breast and the age of the woman. Around $20 \%$ of women aged 50 to 69 years old face the issue of a false-positive result. $3 \%$ of women would conduct a second screening due to a false-positive test and tested for a lumpectomy. This survey accounted for 76 of 100,000 women in Europe. $^{10}$

\section{False-Positive Results}

In this case, the patient is diagnosed with Cancer-Free while she has cancer cells. This leads to ignoring cancer for another two years until it shows symptoms. In this case, the benefit of the mammography is nil since it has not been able to detect malignant cells. Around 28 to $33 \%$ of cases are Interval cancers - overlooked cancer cells through biopsy. Many medical institutes are shifting towards the usage of digital mammography and this has proved to reduce the number of inaccurate results. However, there are still many risks associated with Digital mammography.

\section{Over-diagnosis}

Many studies and researches have proved the harm of being overly diagnosed. The theory discussed the pandemic that affected Japan due to the screening of infants for neuroblastoma. ${ }^{8}$ A small group of 50 infants was screened and this led to the whole country having to conduct screening of infants due to the doubling of the cases from neuroblastoma. This situation was provoked by the development of tumour while being diagnosed. This is how over-diagnosing a disease can trigger the development of unwanted disease. ${ }^{12}$ The same concept is studied in the case of breast cancer cell formation. An individual who develops cells at a slower pace has high risks of developing breast cancer with overdiagnosis ${ }^{8}$. He explains that the process of cells dying, promotes renewal and when x-rayed, some of these cells become infected. If they do not renew quickly, this may cause the cells to become malignant. ${ }^{10}$ Kalager's study in Europe during 1996 and 2005 proved that over-diagnosis promoted breast cancer. Breast Screening programs were introduced in parts of Norway and a few years later, there was a slight increase of 5\% in the cases of breast cancer. The number was compared to other regions in Norway where the biopsy was not practiced and there was no increase in the disease which shows a positive relationship between breast cancer and overdiagnosis. ${ }^{14}$

Overall, the utility of the device is the biggest concern in the detection of cancer, and secondly the risks of radiation caused by x-ray beams. The graph below shows a contrast between the harms against the benefits of mammography (Figure1). It is based on an observational survey from 1,000 women screened every $2^{\text {nd }}$ year for 20 years, starting from their fifties. 


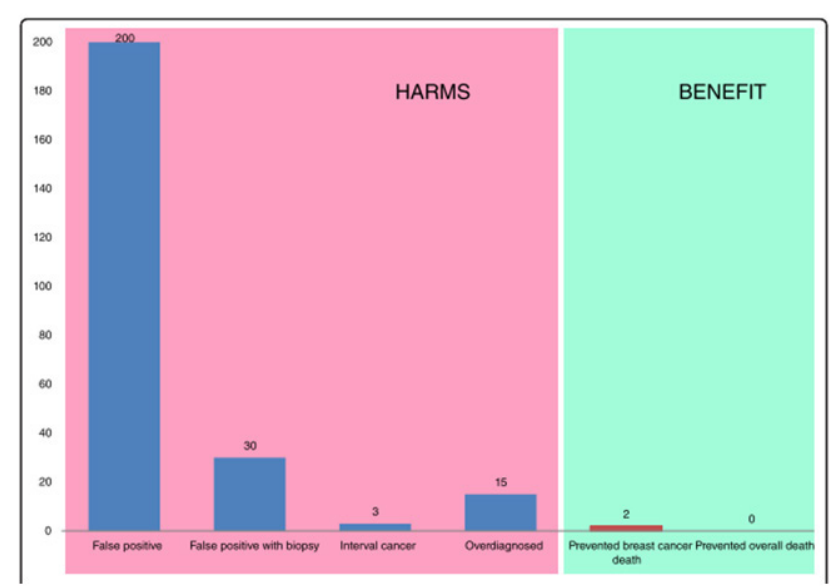

Figure 1: Harms v/s Benefits of Mammography.

Figure 1 describes the possible harms of mammography against the benefits. The statistics are collected from 1,000 women who were screened every two years interval starting at the age of 50, for the next 20 years of their lives. The harms of facing a False Positive result are considerably high and the aftermath of this causes further health and mental complications such as anxiety and depression. Another leading factor to be discussed is over-diagnosis, where $15 \%$ of women have been overexposed to radiation emittance causing the formation of cancerous cells. ${ }^{8}$

\section{MAMMOGRAPHY ALTERNATIVES}

There are more than twelve devices designed for the early detection of breast cancer but only three have been approved to be used by the FDA. The devices are Screen-Film Mammography, Digital Mammography, and Computer-Aided Detection (CAD). The widely used devices are the screen-film and the digital. Many tests were assessed with the following devices and despite its side-effects, the FDA assures that this is the best device for early breast cancer detection. The statement led to further research by the Institute of Medicine and National Research Council (US) Committee on the early detection of Breast Cancer, to identify that mammography does not impact positively on the mortality rate of several women in different countries. Several debates were raised on the effectiveness of the device since countries such as Lebanon, Spain, and Switzerland encountered a massive increase in breast cancer cases after implementing Biopsy. ${ }^{5}$

\section{Computer-Aided Detection}

Computer-Aided Detection uses the same concept as digital mammography except it does not emit radiation. The CAD uses the technology of advanced algorithms based on many thousand cases of breast cancer. The CAD is an extension of digital mammography which becomes useful is the patient's case is suspicious. After the primary detection, the ra- diologist will run $\mathrm{CAD}$ on the imaging received to sense the different tests on the affected areas of the breast. However, as the device is a continuation of Digital Mammography to enhance the imaging, the patient will still need to undergo a biopsy and has potential risks of developing the side-effects of radiation exposure (Computer-Aided Detection (CAD).

\section{Thermal Technology}

The largest surface in humans is the Skin. It is responsible for controlling the temperature of the human body in different conditions such as sweating, freezing, and normal. Hence, the technology of thermal has been introduced to detect the malignant areas of breast cancer. The thermal technology can detect cancer irrespective of the women's age and their breast density. On the other hand, the usage of thermography is completely safe as it is non-invasive and completely painless and more importantly, there is no invasion of harmful radiation ${ }^{6}$.

The science behind thermography is the usage of a thermal infrared camera to picture the released radiation (body warmth) from the human body and hence, re-produce a thermogram that reflects the heat pattern and distribution of the body area. Cancerous are usually, denser, and warmer than surrounding cells that will help to reflect the affected regions. As it is shown in Figure 2, the interpretation of the results will be colour-based where the warmer shows red and the cooler shows blue. The information about the bodily patterns will be collected through sensors placed around the breast. Sensors have to be placed in specific areas for 12 hours to achieve the best results. ${ }^{5}$

\section{EFFECTIVENESS OF A THERMAL SENSOR DEVICE}

The accuracy obtained from a thermal sensor device has proven to be more specific than mammography. Cyrcadia is American produce that innovated the medical industry with the wearable monitor brassiere that enables to detect any abnormalities in the breast area under 12 hours. Until now, twenty-six clinics have adopted the use of the device for the first screening. The device is completely radiationfree and safe to use. Many tests have been done to assess the effectiveness of the device. It has been found that if the sensors are misplaced, this will lead to an error that signals the patient that the device is not working properly. The conditions of use are that the patient must not participate in any activities which may lead to excessive sweating to not bias the results..$^{10,14}$

The Cyrcadia device comes with a sensor-brassiere and software. The software is helpful to read the information gathered by the sensors. A medical practitioner must instruct the patient the usage procedures on their first time and the indi- 
vidual may then carry their test by themselves at the comfort of their home. ${ }^{12}$

\section{UNDERPINNING THEORIES AND RESEARCH MODEL}

The theories studied in this research are from accredited sources namely medical instates for cancer. However, different perspectives on mammography are acquired from Science Direct where many theories are analyzed. Many researchers have identified many factors that trigger the formation of breast cancer such as stress and anxiety. The subjects are highly debatable at this point since no experiment has yet been conducted to find the relationship with cancer formation and stress/anxiety. Theories about how lifestyle impacts on health is another major factor that contributes a lot to the formation of cancerous cells. The continuous unhealthy life practices such as alcohol, fast food, drugs, etc lead to accumulated DNA damage that will manifest in the sort of diseases. These theories are applied in areas of the research to give the reader a broader perspective of the causes of breast cancers. ${ }^{9,13,14}$

The Research Model allows the distinction between the independent factors to the dependent variable. In that context, the different hypotheses of mammography.

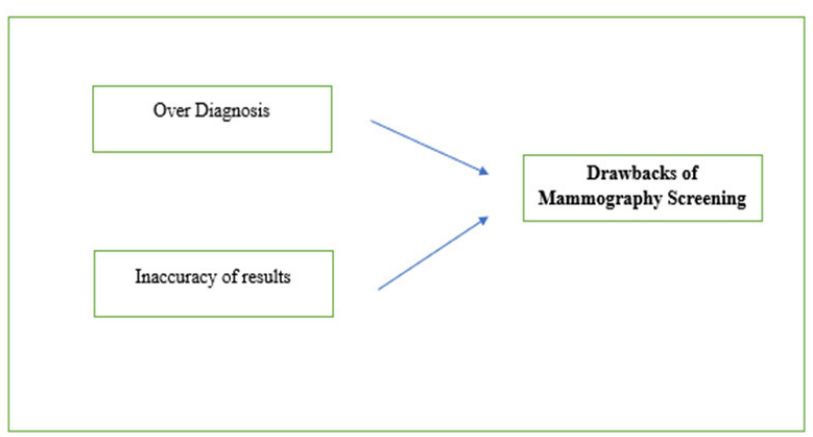

Figure 2: Research Model.

\section{CONCLUSION}

The study evaluates the risks of mammography. To conclude, the evaluation finds mammography to be amongst one of the causes of breast cancer. Over-diagnosis has a direct impact on women under 50 years and can be very risky to their health. Furthermore, inaccuracy of mammography leading to falsepositive/ false-negative results can impact women's mental health and in certain situations aggravate the disease. Hence, the concept of a thermal device for breast cancer screening is well justified being a safer and non-invasive method for screening.

\section{REFERENCES}

1. Breast Cancer Now. Percentage of women going for breast screening falls again. 2020. [online] Available at: https://breastcancernow.org/about-us/news-personal-stories/percentagewomen-going-breast-screening-falls-again [Accessed 26 Feb. 2020].

2. Breast Cancer Screening. 3rd ed. Lyon, France: International Agency for research on cancer, 2016. https://www.ncbi.nlm.nih. gov/books/NBK546558/

3. Buist D, Porter P, Lehman C, Taplin S. White E. Factors Contributing to Mammography Failure in Women Aged 40-49 Years. J Nat Cancer Institute, 2004;96(19):1432-1440.

4. Deniz S, Kurt B, Oguzoncul A. Knowledge, attitudes and behvavior of women regarding breast and cervical cancer i Malatya, Turkey. Plos One 2017; 12(11): e0188571.

5. Eccles S, Oboagye E, Thompson A. Critical research gaps and translational priorities for the successful prevention and treatment of breast cancer. Breast Cancer Research 2013; 15:R92.

6. Laila FU, Afikah NZ, Safiee N, Asnida A, Rafiq M, Ramlee M. Development of A Low-Cost Wearable Breast Cancer Detection Device. 2018 2nd International Conference on BioSignal Analysis, Processing and Systems (ICBAPS).

7. Linktolife.mu. Breast Cancer Screening | Link to Life Mauritius. 2020 [online] Available at: http://www.linktolife.mu/services/ breast-cancer-screening [Accessed 29 Feb. 2020].

8. Rajendran K, Jayabalan M, Thiruchelvam V, Sivakumar V. Feasibility study on data mining techniques in diagnosis of breast cancer. Int J Machine Learn Comp 2019;9(3): 328-333.

9. Sathya M, Madhan S, Jayanthi K. Internet of things (IoT) based health monitoring system and challenges. Int J Eng Tech 2018; $7: 175$.

10. Fletcher S, Elmore J. Mammographic Screening for Breast Cancer. New Eng J Med 2003;348(17):1672-1680.

11. Hanley J, Hannigan A, O'Brien K. Mortality reductions due to mammography screening: Contemporary population-based data. PLoS ONE 2017; 12(12):e0188947.

12. Hendrick R. Radiation Doses and Cancer Risks from Breast Imaging Studies. Radiology 2010;257(1):246-253.

13. Kori S. An Overview: Several Causes of Breast Cancer. Epidemol Int J 2018; 2(1).

14. Løberg M, Lousdal M, Bretthauer M, Kalager M.. Benefits and harms of mammography screening. Breast Cancer Res $2015 ; 17(1)$. 